\title{
Futures-based forecasts: How useful are they for oil price
}

\section{volatility forecasting?}

\author{
Ioannis Chatziantoniou*, Stavros Degiannakis ${ }^{* *, * * *}$, and George Filis ${ }^{* * *, a}$ \\ ${ }^{*}$ Economics and Finance Subject Group, University of Portsmouth, Portsmouth Business School, \\ Portland Street, Portsmouth, PO1 3DE, United Kingdom. \\ ** Department of Economics and Regional Development, Panteion University of Social and Political \\ Sciences, 136 Syggrou Avenue, 17671, Greece. \\ ${ }^{* * *}$ Bournemouth University, Department of Accounting, Finance and Economics, Executive Business \\ Centre, 89 Holdenhurst Road, BH8 8EB, Bournemouth, UK. \\ ${ }^{a}$ Corresponding author's email: gfilis@bournemouth.ac.uk
}

May 13, 2019

\begin{abstract}
Oil price volatility forecasts have recently attracted the attention of many studies in the energy finance field. The literature mainly concentrates its attention on the use of daily data, using GARCH-type models. It is only recently that efforts to use more informative intraday data to forecast oil price realized volatility have been made. Despite all these previous efforts, no study has examined the usefulness of futures-based models for oil price realized volatility forecasting, although the use of such models is extensive for oil price predictions. This study fills this void and shows that futures-based forecasts based on intra-day data provide informative forecasts for horizons that span between 1-day and 66-days ahead. More importantly, these results hold true even during turbulent times for the oil market, such as the Global Financial Crisis of 2007-09 and the oil collapse period of 2014-15.
\end{abstract}

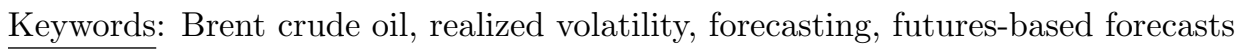

JEL codes: C22, C53, G13, Q47 


\section{Introduction}

Changes in oil prices have numerous repercussions on several aspects of economic activity such as, portfolio management, risk-assessment, financial options' pricing, as well as, macroeconomic policy formulation. In point of fact, to the extent that abrupt oil price changes affect both the financial sector and the real economy, we maintain that oil price volatility could convey valuable information for both policy makers and investors. It follows that, producing accurate forecasts of oil price volatility becomes rather essential.

The important role of oil price volatility forecasting in the decision making process of the aforementioned stakeholders has been highlighted in the works of Cabedo and Moya (2003), Giot and Laurent (2003), Elder and Serletis (2010), Xu and Ouenniche (2012), Silvennoinen and Thorp (2013), Sévi (2014), as well as, Zhang and Zhang (2017), among many others. What is more, the growing interest in accurately predicting oil price volatility stems also from the intense - in recent years - financialization of the oil market. To be more explicit, the years 2004-2005 marked the beginning of a period whereupon commodities started to behave more like financial assets as opposed to physical assets; a fact which practically implies that oil price changes have since been more closely linked to developments in financial markets (see, for example, Vivian and Wohar, 2012; Silvennoinen and Thorp, 2013; Basher and Sadorsky, 2016; Le Pen and Sévi, 2017). Thus, given the mounting importance of oil price volatility forecasting for decision making, developing appropriate forecasting practices, is in fact a challenging field of study.

A common ground shared by all studies on oil price volatility forecasting, as we show below, is that they do not assess the usefulness of the futures-based models. However, authors such as Alquist and Kilian (2010) emphasize the valuable input of the futures market in the estimation of future spot oil prices. In particular, Alquist and Kilian (2010) explain that it is customary for policy institutions such as Central Banks or the International Monetary Fund (IMF) to capture expectations regarding the future spot oil price through the price for oil futures. In fact, there are numerous studies that employ futures-based forecasts to predict spot oil prices ${ }^{1}$. By contrast, as aforementioned, the potential predictive information of the futures market on oil price volatility forecasting has been rather neglected. Thus, the main objective of this study is to fill this void and to investigate whether oil futures price volatility could carry important predictive power over oil spot price volatility.

\footnotetext{
${ }^{1}$ See, inter alia, Coppola (2008); Murat and Tokat (2009); Alquist and Kilian (2010); Chinn and Coibion (2014).
} 
With reference to relevant studies in the field, oil price volatility forecasting dates back to the work of Sadorsky (2006) and Sadorsky and McKenzie (2008). Their work is predicated on daily data and emphasizes GARCH-type models as being the most appropriate in producing accurate forecasts of conditional volatility. At this point, it should be noted that, for their most part, early studies in the field were primarily based on high- to low-frequency data (i.e., daily, weekly or monthly) and GARCH-type models (see, inter alia, Kang et al., 2009; Nomikos and Pouliasis, 2011; Kang and Yoon, 2013), rather than ultra high-frequency data (i.e. intra-day data). What is also common in these studies is the general consensus that univariate GARCH-type models are superior to their multivariate counterparts in forecasting accuracy, regarding mainly 1-day, 5-day, as well as, 10-day ahead forecasts (see, for instance, Agnolucci, 2009; Wei et al., 2010; Arouri et al., 2012; Chkili et al., 2014; Charles and Darné, 2017).

Turning to a point mentioned earlier in connection with alternative information channels, the study by Efimova and Serletis (2014) is the first one to utilize S\&P500 daily returns (i.e., an asset class outside energy markets) towards forecasting oil price conditional volatility; however, the results of the study show that this additional class is not conducive to superior forecasts. On a final note, in as much as forecasting conditional volatility is the main objective, it should be stressed that little attention has been paid to the role of futures prices as a predictor of oil price volatility (exceptions include, Nomikos and Pouliasis, 2011; Kang and Yoon, 2013).

Nonetheless, the ground-breaking work of Andersen and Bollerslev (1998) implies that focus should be put on the realized volatility (RV) instead. Realized volatility considers intra-day returns in order to produce a more accurate measure of daily volatility. It has long been established, especially in the finance literature, that intra-day data can produce more accurate forecasts than daily data (Andersen et al., 2001, 2003, 2005; Hansen and Lunde, 2005; McAleer and Medeiros, 2008; Tay et al., 2009). Despite the fact that the realized volatility measure was introduced three decades ago and that extensive research has been published since then on forecasting realized volatility of stock markets and exchange rates, demonstrating its superior ability (see, among others, Andersen et al., 2003, 2005; Hansen and Lunde, 2005; Bauer and Vorkink, 2011; Busch et al., 2011; Chiriac and Voev, 2011), a very limited number of studies have been only recently conducted in connection with the oil market.

The earliest studies in this line of research belong to Haugom et al. (2014) and Sévi (2014). In particular, Haugom et al. (2014) employ the Heterogeneous AutoRegressive (HAR) model of Corsi (2009) to provide evidence that the inclusion of the oil price implied volatility significantly improves forecasts for the oil price realized volatility. What is more, authors such as Sévi (2014) 
but also more recently Prokopczuk et al. (2016) employ a variety of HAR specifications in order to improve forecasts by further considering volatility jumps. Both authors show that modelling jumps does not lead to more accurate forecasts of realized volatility, implying that the simple version of the HAR model is quite effective for the purposes of forecasting.

A very recent study by Degiannakis and Filis (2017) is also grounded on the HAR framework and provides evidence in support of the argument that considering cross-market information flows (i.e., information from alternative asset classes' volatilities) enhances the predictive accuracy of oil price realized volatility.

With this in mind, we reiterate that the contribution of this study is to investigate the usefulness of futures price volatility as a predictor of oil price volatility. Nevertheless, we also add to the rather scarce strand of the literature, which is associated with the use of ultra high-frequency data (intra-day) for the purpose of oil price realized volatility forecasting.

To do so we employ intra-day data - for the period August 1, 2003 to August 5, 2015 on Brent Crude oil prices for the 1-month, 2-month and 3-month futures contracts, as well as, spot prices of Brent Crude Oil (ICE Futures Europe). As aforementioned, we focus on oil price realized volatility, as this can be constructed by ultra-high frequency (i.e., intra-day data) and we concentrate on short- and medium-run forecasts; that is, forecasts for 1-day to 66-days ahead.

Main findings indicate that futures-based forecasts (especially the models based on the 1month futures contracts) can be accurate predictors of oil price volatility, significantly improving the forecasting accuracy of the no-change forecasts by more than $70 \%$. Furthermore, based on the Model Confidence Set, the futures-based models that exhibit the highest predictive accuracy are those that either relax the proportionality restriction or both the unbiasedness assumption and the proportionality restriction. We further show that futures-based forecasts exhibit very high directional accuracy that significantly exceeds the $68 \%$ level for the full sample estimation and the $90 \%$ level during turbulent periods. On a final note, results are suggestive of the fact that futures-based forecasts are also very useful for oil price volatility predictions during crisis periods (e.g., Global Financial Crisis of 2007-09 and the oil collapse period of 2014-15).

The remainder of the paper is structured as follows. Section 2 presents the forecasting models and data used in this study. Empirical results are presented in Section 3, before Section 4 concludes our analysis. 


\section{Data and forecasting methodology}

\subsection{Data description}

In this study we use tick-by-tick data of the spot prices of the Brent Crude Oil (ICE Futures Europe), as well as, for the 1-month, 2-months and 3-months futures contracts expirations. The choice of these three maturities is justified by the fact that they represent the most liquid futures contracts. All data are obtained from TickData. The period of our study spans from August 1, 2003 to August 5, 2015 and it is dictated by the availability of intra-day data for the Brent Crude oil futures contracts. The tick-by-tick data are used to construct the daily realized volatilities of the spot, as well as, the futures oil prices. The continuous time series for the futures prices have been constructed using the most active portion of each contract. We roll to the next contract based on the most liquid future contract at any given month ahead, i.e. when the day-session's tick volume of the current contract is lower than the daily tick volume of the following month contract.

Before the construction of the daily realized volatilities, there are four important considerations to be made. First, following Andersen et al. (2001, 2003, 2005), this paper considers that a full trading day spans between 21:01 GMT the night before until 21:00 GMT that evening. Second, we exclude from our sample series the fixed and moving holidays, e.g. Christmas, Martin Luther King day, Washington's Birthday, Good Friday, Easter Monday, Memorial day, July 4th, Labour day and Thanksgiving and the day after. Third, we remove any oil prices that are reported between Friday 21:01 GMT until Sunday 21:00 GMT. The final consideration is related to the sampling scheme.

There are three sampling schemes, namely, calendar time sampling, transaction time sampling and tick time sampling. In calendar time sampling, the prices are sampled at regular intervals in calendar time, e.g. every 5 minutes. In transaction time sampling the prices are sampled at every specific number of transactions, whereas, in tick time sampling the prices are recorded at each price change. Oomen (2006) shows that sampling in transaction time is generally superior to sampling in calendar time in that it leads to a lower mean squared error of the realized volatility measure. Additionally, Griffin and Oomen (2008) provide evidence that the realized volatility measure constructed in tick time sampling is superior compared to those constructed in transaction time sampling, when the level of noise or the number of ticks is low. However, as Patton (2011) concludes, although tick time sampling leads to more accurate realized variance estimation compared to the calendar time sampling, this difference disappears as 
we move closer to the most recent time periods (for instance in his paper these differences in the two sampling schemes tend to be negligible as the sub-sample included information closer to 2007). Having considered the aforementioned papers, we have decided to adopt the calendar sampling due to (i) the use of recent data, (ii) the fact that the asymptotic properties of transaction time sampling schemes are clearly less well-known (see, for example, Sévi, 2014) and require pre-specific assumptions about the microstructure of the market or the stochastic process of the assets (see, for instance, Patton, 2011), (iii) comparability of the findings with the models of the existing literature and finally (iv) data availability to the wider community of researchers (the majority of researchers do not have tick-by-tick data at their disposal).

\subsection{Realized volatility construction}

According to Andersen and Bollerslev (1998) the daily realized volatility is estimated based on the sum of squared intra-day returns:

$$
R V_{t}^{(\tau)}=\sqrt{\sum_{j=1}^{\tau}\left(\log P_{t_{j}}-\log P_{t_{j-1}}\right)^{2}}
$$

where $P_{t_{j}}$ denotes the observed oil prices (either spot or futures) at day $t$, and $\tau$ refers to the equidistant intra-day time intervals. However, in order to construct the realized volatility according to Eq. 1, we need to identify the optimal intra-day time intervals, $\tau$. According to Andersen et al. (2006) the optimal number of intra-day time intervals is the one that minimizes the measurement bias, which is inserted in the estimation due to microstructure frictions. To find the optimal $\tau$, we decompose the inter-day variance into the intra-day variance and the intra-day autocovariances, as follows:

$$
\left(\log P_{t}-\log P_{t-1}\right)^{2}=\left(R V_{t}^{(\tau)}\right)^{2}+2 \sum_{j=1}^{\tau-1} \sum_{i=j+1}^{\tau}\left(\log P_{t_{i}}-\log P_{t_{i-1}}\right)\left(\log P_{t_{i-j}}-\log P_{t_{i-j-1}}\right)
$$

where the latter part to denote the measurement bias, with $\left(\log P_{t_{i}}-\log P_{t_{i-1}}\right)\left(\log P_{t_{i-j}}-\right.$ $\left.\log P_{t_{i-j-1}}\right)=0$, for $j \neq 0$. The optimal $\tau$ is the one that minimizes this bias. In our case $\tau=23$ minutes.

Furthermore, motivated by Hansen and Lunde (2005), we proceed to the adjustment of the realized volatility with the close-to-open volatility (Eq. 3), given that even when markets are closed, information still flows. 


$$
R V_{\text {adjusted }, t}^{(\tau)}=\sqrt{\omega_{1}\left(\log P_{t_{1}}-\log P_{t-1_{\tau}}\right)^{2}+\omega_{2} \sum_{j=2}^{\tau}\left(\log P_{t_{j}}-\log P_{t_{j-1}}\right)^{2}},
$$

where the weights $\omega_{1}$ and $\omega_{2}$ are the optimal weights that minimise the variance of the realized volatility. Finally, we estimate the annualised daily realized volatility $(A R V)$, as follows:

$$
A R V_{\text {adjusted,t }}^{(\tau)}=\sqrt{252} \times R V_{\text {adjusted, },}^{(\tau)}
$$

Figure 1 exhibits the annualised daily realized volatilities of the spot and futures oil prices, whereas Table 1 presents the descriptive statistics of our series.

[Insert Figure 1 about here]

[Insert Table 1 about here]

From Figure 1 we observe that the behaviour of all realized volatilies are similar over time, although one noticeable difference is the fact that as we are progressing from the spot realized volatility to the 3 -month ahead, its magnitude decreases. This is particularly evident during the Global Financial Crisis period of 2007-09, where the peak in the volatilities is observed (see, max. values in Table 1). Furthermore, from Table 1 we notice that all volatilites have excess kurtosis and positive skewness, as expected. Finally, all our series are stationary, as shown by the ADF test. Next, we proceed with the description of our forecasting models.

\subsection{Naive forecasting models}

The random walk (RW) with drift assumes that the best $s$-steps-ahead forecast of the annualised logarithmic realized volatility of the spot oil price, i.e. $\log \left(A R V_{\text {spot,adjusted,t+s }}^{(\tau)}\right)$, is the most recently available price volatility plus the average logarithmic growth rate, $\phi_{0}^{(t)}+$ $\log \left(A R V_{\text {spot,adjusted,t }}^{(\tau)}\right)$. Hence, based on the fully uninformative relationship of $E\left(\log \left(A R V_{\text {spot,adjusted,t+s }}^{(\tau)}\right) \backslash\right.$ $\left.I_{t}\right)=\phi_{0}^{(t)}+\log \left(A R V_{\text {spot, adjusted, } t}^{(\tau)}\right)+e_{t}$, where $I_{t}$ is the information set available at time $t$ and $e_{t}$ denotes the standard normally distributed error term, the benchmark for comparing the forecasts is:

$$
A R V_{\text {spot }, \text { adjusted }, t+s}^{(\tau)}=\exp \left(\phi_{0}^{(t)}+\log \left(A R V_{\text {spot, adjusted }, t}^{(\tau)}\right)\right)
$$

We consider the logarithmic transformation of $A R V_{\text {spot,adjusted, },}^{(\tau)}$ as in the majority of the studies that model the realized volatility. 
Due to the autocorrelated structure of the volatility, we decided to construct naive forecasts of the annualised realized volatility of the spot oil price form an autoregressive structure of 1st order $(\mathrm{AR}(1))$, as well: $E\left(\log \left(A R V_{\text {spot,adjusted,t+s }}^{(\tau)}\right) \backslash I_{t}\right)-\phi_{0}^{(t)}=\phi_{1}^{(t)}\left(\log \left(A R V_{\text {spot, }, \text { adjusted }, t}^{(\tau)}\right)-\phi_{0}^{(t)}\right)+e_{t}$, where $\phi_{0}^{(t)}$ and $\phi_{1}^{(t)}$ denote the OLS estimates obtained from recursive regressions based on the data available at time $t$. The AR(1) model provides us with the $s$-steps-ahead forecasts computed as:

$$
A R V_{\text {spot,adjusted,t+s }}^{(\tau)}=\exp \left(\phi_{0}^{(t)}\left(1-\phi_{1}^{(t)}\right)+\phi_{1}^{(t)} \log \left(A R V_{\text {spot,adjusted }, t}^{(\tau)}\right)\right)
$$

\subsection{Futures-based forecasting models}

In line with the approach we followed in Section 2.3 for the random walk model, we assume that the best $s$-steps-ahead forecast of the annualised realized volatility of the spot oil price, i.e. $A R V_{\text {spot,adjusted,t+s }}^{(\tau)}$, is the most recently available futures oil price volatility with $h$ months to maturity, i.e. $A R V_{F^{(h)}(\tau)}^{(\tau)}$ adjusted,t . Hence, we assume that:

$$
E\left(\log \left(A R V_{\text {spot, adjusted,t+s }}^{(\tau)}\right) \backslash I_{t}\right)=\log \left(A R V_{F^{(h)}, \text { adjusted, } t}^{(\tau)}\right)+e_{t}
$$

Thus, the $s$-steps-ahead forecasts are computed as:

$$
A R V_{\text {spot,adjusted,t+s }}^{(\tau)}=A R V_{F^{(h)}, \text { adjusted, }}^{(\tau)}
$$

Alternatively, we model the ratio of futures oil price volatility and current spot oil price volatility, i.e. futures volatility spread, as a rational predictor of the volatility of spot oil price at time $t+s$ :

$$
\log \left(\frac{A R V_{\text {spot,adjusted, } t}^{(\tau)}}{A R V_{\text {spot }, \text { adjusted, },-s}^{(\tau)}}\right)=\phi_{0}^{(t)}+\phi_{1}^{(t)} \log \left(\frac{A R V_{F^{(h)}, \text { adjusted, } t}^{(\tau)}}{A R V_{\text {spot, adjusted, },-s}^{(\tau)}}\right)+e_{t} .
$$

The $s$-steps-ahead forecast of the annualised realized volatility of the spot oil price is computed as:

$$
A R V_{\text {spot, adjusted, } t+s}^{(\tau)}=A R V_{\text {spot, adjusted }, t}^{(\tau)} \exp \left(\phi_{0}^{(t)}+\phi_{1}^{(t)} \log \left(\frac{A R V_{F^{(h)}, \text { adjusted, }, t}^{(\tau)}}{A R V_{\text {spot }, \text { adjusted }, t}^{(\tau)}}\right)\right)
$$


Additionally, the equation (9) is also estimated separately for $\phi_{1}^{(t)}=1$ and for $\phi_{0}^{(t)}=0^{2}$, such as that:

$$
\log \left(\frac{A R V_{\text {spot,adjusted,t }}^{(\tau)}}{A R V_{\text {spot, adjusted, } t-s}^{(\tau)}}\right)=\phi_{0}^{(t)}+\log \left(\frac{A R V_{F^{(h)}, \text { adjusted, },}^{(\tau)}}{A R V_{\text {spot, adjusted,t-s }}^{(\tau)}}\right)+e_{t}
$$

and

$$
\log \left(\frac{A R V_{\text {spot,adjusted, },}^{(\tau)}}{A R V_{\text {spot, }, \text { adjusted, },-s}^{(\tau)}}\right)=\phi_{1}^{(t)} \log \left(\frac{A R V_{F^{(h)}, \text { adjusted }, t}^{(\tau)}}{A R V_{\text {spot, }, \text { adjusted }, t-s}^{(\tau)}}\right)+e_{t} .
$$

We do so in order to assess separately (i) the fact that the futures volatility spread may be a biased predictor (i.e. hence the restriction that $\phi_{1}^{(t)}=1$ and $\phi_{0}^{(t)}$ is allowed to obtain any value) and (ii) the proportionality restriction (i.e. $\phi_{0}^{(t)}=0$, whereas $\phi_{1}^{(t)}$ remains unrestricted).

In eqs. $7-12, F$ refers to the futures contracts of the Brent crude oil prices and $h$ denotes the futures contracts maturities, i.e. $h=1,2,3$ months. In the tables and figures we denote $C O 1$, $\mathrm{CO} 2$ and $\mathrm{CO} 3$ the futures contracts with 1 - to 3 -months maturities. Furthermore, $s$ denotes the $s$-steps-ahead forecasting horizon, i.e. $s=1,2,3, \ldots, 66$ days. The choice of the forecasting horizons reflects (i) the nature of the futures market, which are rather short to medium-run oriented and (ii) the requirements of forecasting volatility users such as, traders, industry users and policy makers. Finally, $\phi_{0}^{(t)}$ and $\phi_{1}^{(t)}$ denote the least-squares estimates which are obtained from recursive regressions.

\subsection{Forecast evaluation}

Following Degiannakis and Filis (2017) we use an initial sample period of $\tilde{T}=1000$ days and we use the remaining $\breve{T}=2076$ for our out-of-sample forecasting period. The $\tilde{T}=1000$ is justified by the fact that (i) we require a large sample size for the estimation of the models and (ii) we need our initial sample to stop before the GFC of 2007-09. This allows us to include the recession period in our out-of-sample period.

We should also note that for robustness, we further apply a structural breakpoint test as another tool for choosing our initial sample period. Pesaran and Timmermann (2007) maintain that the in-sample period should be the pre-break period. There are various structural breakpoint tests (e.g. Multiple structural break test of Bai and Perron (1998, 2003) or the modified iterated cumulative sum of squares (ICSS) algorithm of Sansó et al. (2004)). In this paper we

\footnotetext{
${ }^{2}$ Note that for $\phi_{0}^{(t)}=0$ and $\phi_{1}^{(t)}=1$, eq. 9 equals to eq. 7.
} 
use the Bai-Perron test, which shows that there is one breakpoint on the 1427th observation, or the 16th March 2009. This date is not included within our initial sample period $\tilde{T}$.

For the first out-of-sample forecasts for 1-day to 66-days ahead, we use the initial sample period $\tilde{T}=1000$. For each subsequent forecast, we use a rolling window approach with fixed length of 1000 days. The forecasting accuracy of the models illustrated in Sections 2.3 and 2.4 are initially evaluated using two well established evaluation functions, namely the Mean Squared Prediction Error (MSPE) and the Mean Absolute Prediction Error (MAPE).

We further employ the newly established Model Confidence Set (MCS) procedure by Hansen et al. (2011), which identifies the set of the best models, as these are defined in terms of a specific loss function, without an a priori choice of a benchmark model, based on the two aforementioned loss functions. Let $L_{i, s}$ to denote the loss function of model $i$ at any $s$-steps-ahead forecasting horizon, and $d_{i, j, s} L_{i, s}-L_{j, s}$ the evaluation differential. Then, the null hypothesis of the MCS is $H_{0, M}: E\left(d_{i, j, s}\right)=0$, for $i, j \in M, M \subset M^{0}$ and it is tested against the alternative $H_{1, M}: E\left(d_{i, j, s}\right) \neq 0$, for some $i, j \in M$. Finally, we also consider the directional accuracy of our models as an additional forecasting evaluation technique. The directional accuracy reports the proportion of the out-of-sample days that a model correctly predicts the direction (up or down) of the volatility movement. For technical details of the MCS test and the directional accuracy please see Hansen et al. (2011).

\section{Empirical Results}

\subsection{Full sample forecasting analysis}

The results for the full out-of-sample period are reported in Table 2, as ratios relative to the no-change forecast. A score below 1 denotes that the forecasts of the $\operatorname{AR}(1)$ and the futuresbased models outperform these of the RW. In Table 3 we show the directional accuracy of our competing models.

[Insert Table 2 about here]

[Insert Table 3 about here]

As shown in Table 2, none of the futures-based forecasts can outperform the no-change forecasts in the 1-day ahead horizon. The only exceptions are the CO1eq12 and CO2eq12, which marginally performs better compared to the no-change forecast, yet based only on the 
MAPE loss function. By contrast, the AR(1) model is the best performing model for the 1-day ahead forecasts.

Focusing now at each futures contracts maturity, we show that the models which consider the futures volatility as the best predictor of the future value of spot volatility (i.e. eq. 7) are not performing well at any forecast horizon, with maximum predictive gains to be about $1 \%$ at the 22-, 44- and 66-days ahead forecasting horizon. When we relax the unbiasedness assumption (i.e. eq. 11) we notice that the predictive gains start to increase rather materially after the 5 -days ahead horizon and reach the levels of $62 \%$ (for the CO1eq11 based on the MSPE) at the 66-days ahead. Significant predictive gains also hold for the futures-based model of eq. 11, using the futures contracts with 2- and 3-months maturity (i.e. CO2eq11 and CO3eq11), although not of the same magnitude. More importantly, we show that when we relax the proportionality restriction (eq. 12), as well as, both the unbiasedness assumption and proportionality restriction (eq. 9) then the predictive gains are evident at all futures maturities for all horizons after the 5-days ahead. In particular, the predictive gains range between the levels of $39 \%$ and $72 \%$, at the 5 and 66-days ahead, respectively, based on the MSPE, with the highest predictive accuracy to be observed with CO1eq12.

Even more, it is evident from the MCS test, that models based on eqs. 9 and 12, using the volatility of the futures contracts with 1-month maturity (CO1eq9 and CO1eq12), are always included in the set of the best performing models at each forecasting horizon from 5-days ahead onwards. Nevertheless, as the forecasting horizons extends further out we observe that the CO2eq9 and CO2eq12 for the 22- and 44-days ahead, as well as, the CO3eq9 and CO3eq12 for the 66-days ahead, are also included in the set of the best performing models.

This is a rather important finding, which suggests that futures-based forecasts do exhibit significant predictive power, especially compared to the no-change forecast and the AR(1). Thus, we argue that despite the fact that Alquist and Kilian (2010) show that oil futures prices are not good predictors for the future spot price of oil, this does not hold for oil price volatility forecasts. The evidence that future-based models of eqs. 9 and 12 provide the best predictive accuracy stems from the fact that they relax either the proportionality restriction or both the unbiasedness assumption and proportionality restriction. More specifically, the slope of the future price volatility has the capability to provide predictive information which is related to market participants' belief of the future spot price volatility and thus models that incorporate this information are expected to produce more accurate forecasts.

To emphasize this further we show the recursive estimates of the coefficient $\phi_{1}^{(t)}$ over the 
out-of-sample forecasting period (see Figure 2). It is clear that in almost the whole period the coefficient $\phi_{1}^{(t)}$ is significantly lower than one. The only exception is the period towards the end of 2014. Hence, Figure 2 provides additional evidence on the importance of relaxing the proportionality restriction in the futures-based models.

\section{[Insert Figure 2 about here]}

Turning to the directional accuracy of our competing models, Table 3 suggests that all models of all three futures maturities exhibit improved directional accuracy relative to the no-change forecast, which is also significant based on the Pesaran and Timmermann (2009) test. The directional accuracy ranges approximately between $63 \%$ and $69 \%$ and once again the highest predictive accuracy across all horizons is achieved by the futures-based models of eqs. 9 and 12 . Another interesting observation is that as the forecasting horizon increases the higher directional accuracy is obtained by the volatility of the futures contracts with the longest maturity.

Overall, these findings imply that if oil volatility forecast users are primarily interested in the volatility value per se, then they should rely on futures-based models that (i) relax the proportionality restriction and (ii) primarily consider the information extracted from the volatility of the futures contracts with 1-month expiration. By contrast, if these end-users are primarily interested in the direction of the volatility, then they should also consider the volatility of the futures contracts with shorter or longer maturities, depending on their forecasting horizon needs.

Scatterplots in Figure 3 provide a visual representation of the relationship between actual and predicted oil price volatility. The columns correspond to the different forecasting horizons, whereas the first, second and third row compare the spot oil price volatility against the nochange forecast, the $\operatorname{AR}(1)$ model and the forecasts from eq. 12, respectively. It is clear from these scatterplots that the forecasts generated from eq. 12 are the most accurate, and this holds not only for low levels of volatility but for the higher values, as well.

\section{[Insert Figure 3 about here]}

The evidence from this section is in favour of the usefulness of the futures-based forecasts, and in particular eqs. 9 and 12, in predicting oil price realized volatility at all horizons between 5- and 66-days ahead. Nevertheless, our forecasting period includes the Global Financial Crisis period of 2007-09, as well as, the period of the Brent crude oil collapse in 2014-15. In the former case, oil prices fluctuated from an unprecedented spike of $\$ 145$ to a sharp drop at about $\$ 30$ per 
barrel, whereas in the latter period oil price collapsed from the levels of $\$ 115$ to an 11-year low of less than $\$ 37$. Thus, in the next sections we evaluate the predictive accuracy of the futuresbased forecasts in extreme conditions, focusing on the Global Financial Crisis period and the oil collapse period of 2014-15.

\subsection{Forecasting performance during extreme conditions}

Starting from the Global Financial Crisis period, Tables 4 and 5 report the forecast evaluations and directional accuracy, respectively. Based on Table 4 we argue that the results remain qualitatively similar with those presented in the full out-of-sample forecasting period (see, Table 2). Put it simply, once again models based on eqs. 9 and 12 are demonstrating the higher predictive accuracy. Having said this, the other models also provide predictive gains, relatively to the no-change forecast, however, they are not of the same magnitude. More specifically, eqs. 9 and 12 are able to provide forecasting gains that reach the levels of almost $83 \%$ in the 66 -days ahead horizon (see, CO2eq9 in Table 4), based on the MSPE loss function, which is rather impressive. The MCS test also confirms that in the set of the best models with equal predictive accuracy are based on the eqs. 9 and 12, at all horizons. Finally, we should once again highlight the fact that until the 10-days ahead horizon, it is only the futures volatility with 1 month maturity that generates the most accurate forecasts, whereas for the 22-, 44- and 66-days ahead horizons the additional futures contracts maturities (CO2 and CO3) are also included in the set of models with equal predictive accuracy.

\section{[Insert Table 4 about here] \\ [Insert Table 5 about here]}

Results are also very encouraging even when we consider the directional accuracy of the models. In particular, the direction accuracy of the futures-based forecasts is very high and reaches the levels of more than $93 \%$ in the 66-days ahead horizon (see, CO1eq9 and CO1eq12). We should highlight here that these directional accuracy scores are even higher compared to the full out-of-sample period, as shown in Table 3.

We finalize our analysis through the investigation of the predictive and directional accuracy of our competing models during the oil price collapse period of 2014-15. The results are shown on Tables 6 and 7 .

[Insert Table 6 about here] 
[Insert Table 7 about here]

Avoiding any unnecessary repetition, we maintain that the results in this section corroborate those that have already been analysed so far and thus this section does not demand for further analysis. One observation that is important to highlight is that the fact that directional accuracy (see, Table 7) is further increased in this period and exceeds the $96 \%$ level (CO1eq9 and CO1eq12).

Overall, these findings suggest that futures-based forecasts are also useful for forecasting oil price volatility even during turbulent periods.

\section{Conclusions}

In recent years, oil price volatility has rather gained much prominence as a potential key determinant of economic developments. In fact, it has numerous ramifications both for the real economy and the financial sector. Aside from macroeconomic policy decision making, changes in the price of oil may be closely linked to portfolio management and assets' pricing, thereby affecting investors, as well. Subsequently, developing models that produce accurate forecasts of oil price volatility has recently become a rather crucial field of study. Nonetheless, there are no studies to apply futures-based forecasting of oil price volatility. By contrast, existing literature utilizes futures-based forecasting only to the extent that oil prices as such can be predicted. In addition, for their most part, current studies do not consider ultra high-frequency (i.e., intra-day) data for the purpose of oil price realized volatility forecasting.

In this study we fill the said voids by examining the usefulness of futures price volatility as predictors of spot oil price realized volatility. We employ tick-by-tick data for Brent Crude Oil spot prices and for the 1-month, 2-months and 3-months futures contracts, to show that, futures-based forecasts of oil price realized volatility significantly outperform both no-change and $\operatorname{AR}(1)$ forecasts. The period of analysis extends from August 1, 2003 to August 5, 2015, while, the forecast horizons of the study span between 1-day and 66-days ahead, reflecting not only the relatively short-to-medium run nature of the futures market, but also, the rather perishable utility of the volatility forecast per se.

Our findings, based on two standard loss functions, the directional accuracy and the MCS test, show that, futures-based models are indeed useful in forecasting spot oil price realized volatility for all horizons. More specifically, we provide evidence that there are particularly highly significant predictive gains to be realized upon relaxing both the unbiasedness assumption and 
the proportionality restriction that were initially imposed on our futures-based models. What is more, our findings suggest that if oil price volatility users are primarily interested in the forecast of the actual volatility value, then they should rely on futures-based models that (i) relax the proportionality restriction and (ii) primarily consider the information extracted from the volatility of the futures contracts with 1-month expiration. On the other hand, if volatility users are primarily interested in the future direction of the volatility, then they should not only consider the aforementioned futures-based models but also, depending on their forecasting horizon requirements, they need to consider the models with shorter or longer maturities of the futures contracts.

On a final note, findings remain robust even in the light of two major events that fall within our forecasting period; that is, the Global Financial Crisis of 2007-09 and the oil price collapse in 2014-15. This is particularly important as, the forecasting models that we propose in this study, can produce accurate predictions of oil price realized volatility even during turbulent and uncertain periods.

Having documented the usefulness of future-based forecasts for oil price realized volatility forecasting, future studies could concentrate on evaluating their performance against other stateof-the-art econometric frameworks, such as the HAR-RV models. Even more, the evaluation of the future-based forecasts using both longer forecasting horizons, as well as, objective-based loss functions is an interesting avenue for further research. For instance, ranking our forecasting models based on a trading or hedging exercise (e.g. Value-at-Risk) would be important to investors, whereas, an evaluation of such forecasts based on their predictive gains on macroeconomic variables (e.g. inflation or GDP) would be deemed necessary for policy makers.

\section{Acknowledgements}

We would like to thank the editor (Richard S.J. Tol) and two anonymous referees for their invaluable comments and suggestions on a previous version of this paper. Stavros Degiannakis and George Filis acknowledge the financial support from the the European Union's Horizon 2020 research and innovation programme, which has funded them under the Marie Sklodowska-Curie grant agreement No. 658494. Part of this paper was completed while Dr. Chatziantoniou was employed by Webster Vienna Private University in Austria. The usual disclaimer applies. 
Figure 1: Brent crude oil price realized volatilities at different futures contracts' maturities
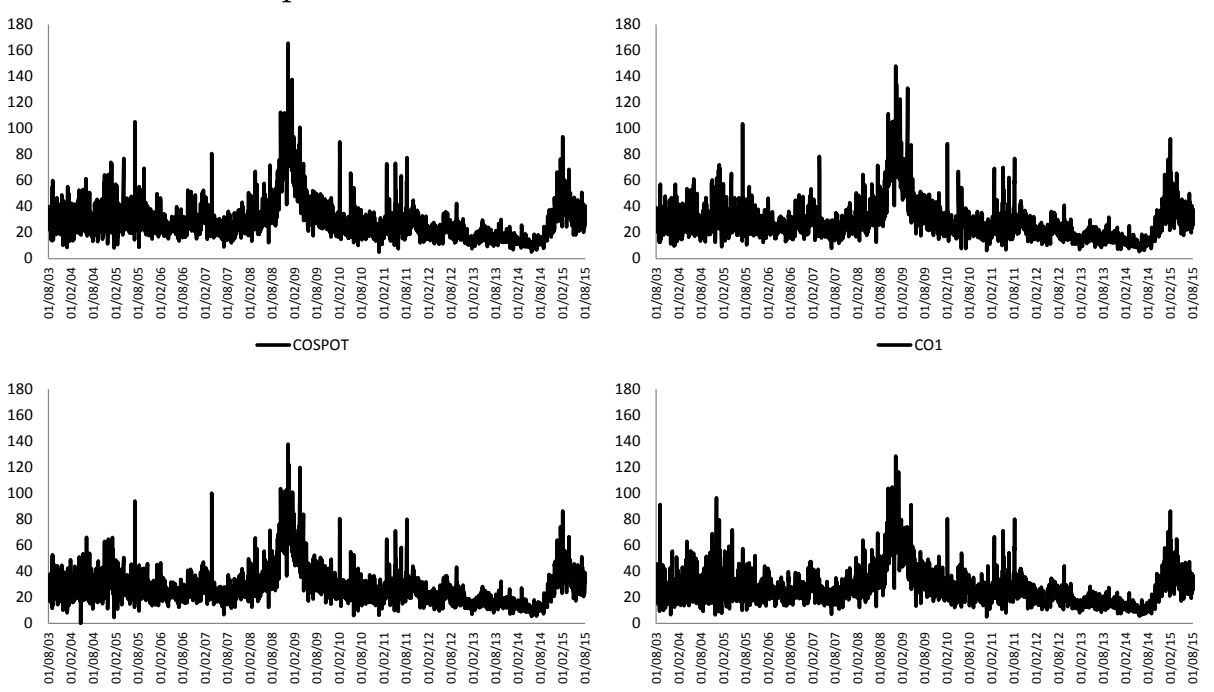

Note: COSPOT denotes the spot oil price realized volatility, whereas $C O 1, C O 2$ and $C O 3$ denote the volatilities of the futures oil prices with 1-month, 2-months and 3-months maturities, respectively. 
Figure 2: The coefficient $\phi_{1}^{(t)}$ from the futures-based model of eq. 12 over the out-of-sample period: $18 / 07 / 2007-05 / 08 / 2015$

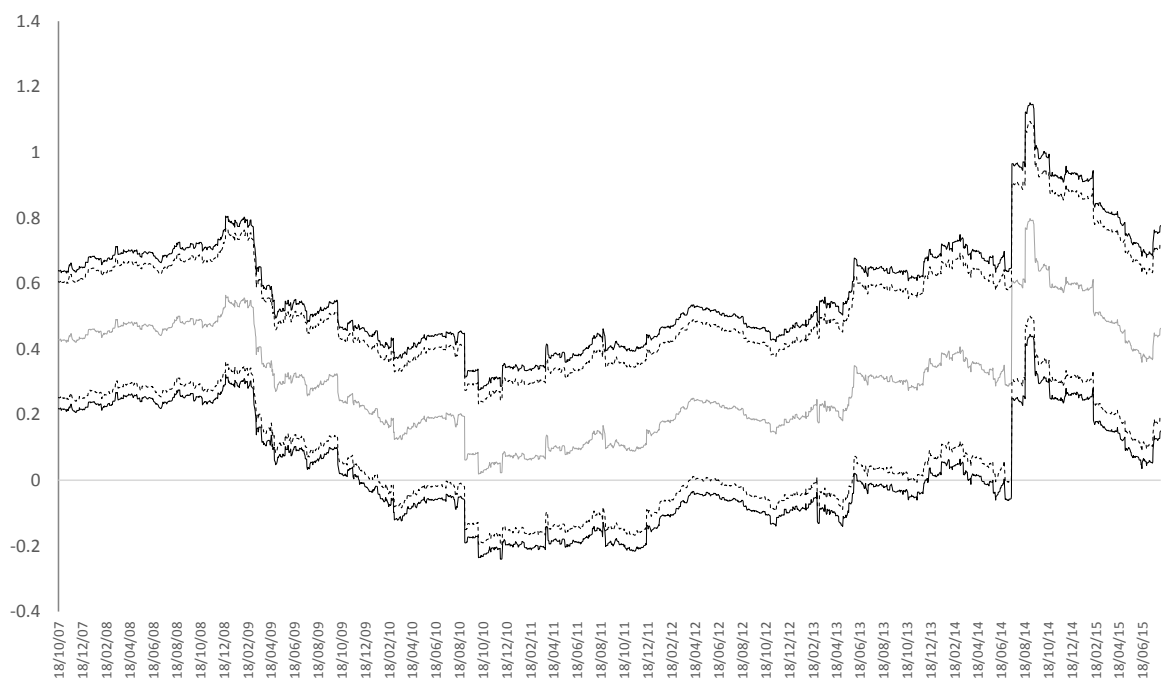

Note: The grey line shows the estimated values of the $\phi_{1}^{(t)}$ coefficient over the out-of-sample forecasting period. The solid (dashed) black lines denote the $95 \%$ (90\%) confidence intervals. 


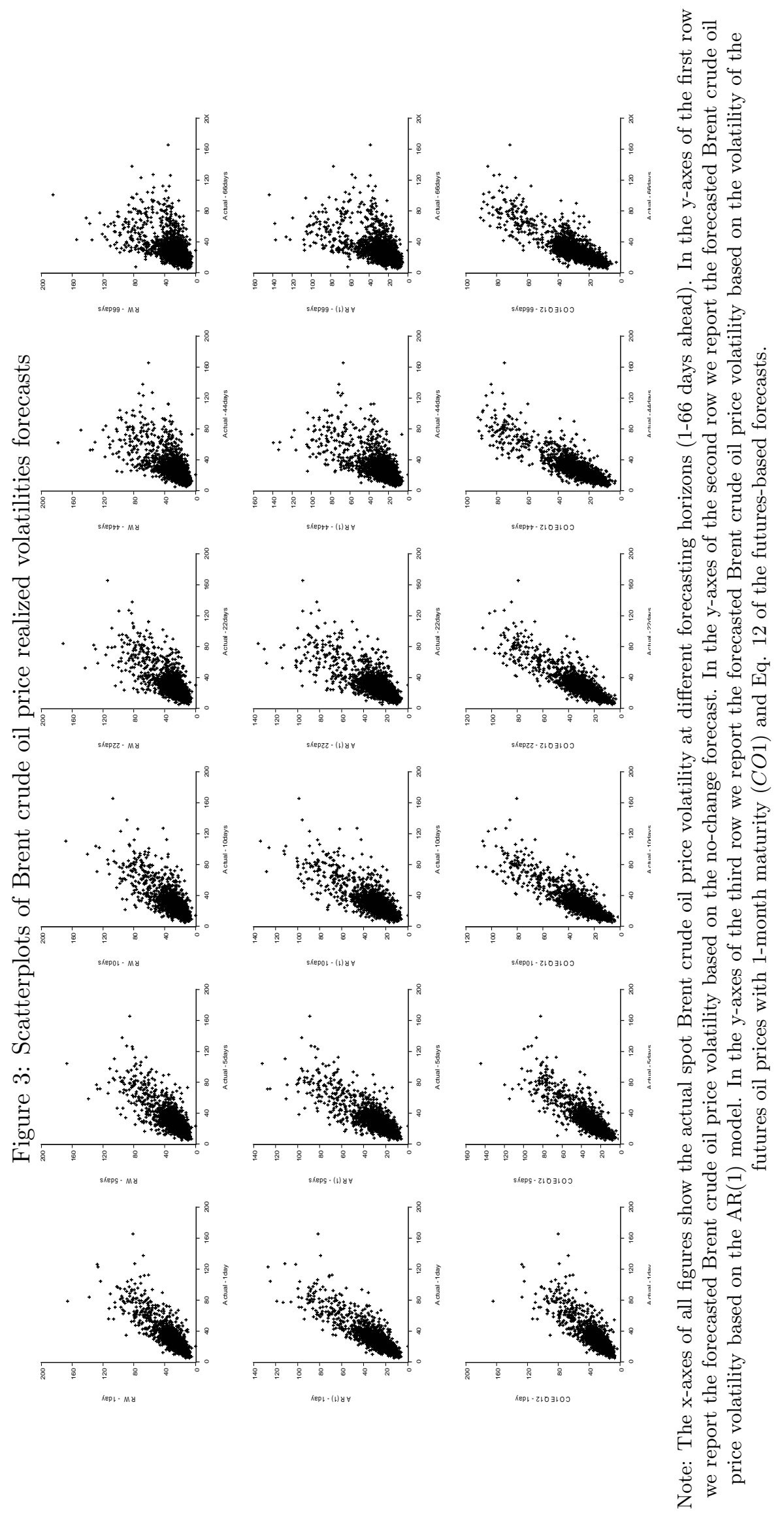


Table 1: Descriptive statistics Brent crude oil price realized volatilities at different futures contracts' maturities

\begin{tabular}{lcccccccccc}
\hline \hline & Mean & Median & Max & Min & Std. Dev. & Skewness & Kurtosis & Jarque-Bera & ADF & Obs. \\
\cline { 2 - 10 } COSPOT & 28.646 & 25.501 & 165.555 & 4.941 & 15.053 & 2.357 & 12.089 & $13436.890^{*}$ & $-3.653^{*}$ & 3076 \\
CO1 & 27.995 & 25.031 & 148.016 & 5.387 & 14.654 & 2.350 & 11.890 & $12959.460^{*}$ & $-3.710^{*}$ & 3076 \\
CO2 & 27.488 & 24.734 & 137.759 & 4.341 & 14.135 & 2.212 & 10.844 & $10395.710^{*}$ & $-3.832^{*}$ & 3076 \\
CO3 & 27.017 & 24.298 & 128.540 & 4.968 & 13.621 & 2.071 & 9.936 & $8363.190^{*}$ & $-3.752^{*}$ & 3076 \\
\hline \hline
\end{tabular}

Notes: * denotes significance at the $1 \%$ level. COSPOT denotes the spot oil price realized volatility, whereas $C O 1$, $\mathrm{CO} 2$ and $\mathrm{CO} 3$ denote the volatilities of the futures oil prices with 1-month, 2-months and 3-months maturities, respectively. The ADF test has been estimated using 13 lags. The ADF regression with a constant is estimated, since the alternative hypothesis is that volatility is stationary above a non-zero mean. 
Table 2: Forecasting oil price volatility - full out-of-sample period. Evaluation period: $18 / 07 / 2007-05 / 08 / 2015$.

\begin{tabular}{|c|c|c|c|c|c|c|}
\hline Days ahead & 1-day & 5-days & 10-days & 22-days & 44-days & 66 -days \\
\hline & \multicolumn{6}{|c|}{ MSPE ratio } \\
\hline $\operatorname{AR}(1)$ & $0.7957^{*}$ & 0.8348 & 0.8629 & 0.9063 & 0.9407 & 1.0067 \\
\hline CO1eq7 & 1.0442 & 1.0024 & 1.0043 & 0.9987 & 0.9888 & 0.9839 \\
\hline CO1eq9 & 1.0078 & $0.6199 *$ & $0.5495^{*}$ & $0.4588^{*}$ & $0.3532^{*}$ & $0.2818^{*}$ \\
\hline CO1eq11 & 1.0698 & 0.7808 & 0.7245 & 0.6190 & 0.4722 & 0.3778 \\
\hline CO1eq12 & 0.9951 & $0.6132 *$ & $0.5420^{*}$ & $0.4523^{*}$ & $0.3472^{*}$ & $0.2773^{*}$ \\
\hline CO2eq7 & 1.0178 & 1.0059 & 0.9904 & 0.9749 & 0.9605 & 0.9546 \\
\hline CO2eq9 & 1.0993 & 0.6735 & $0.5750 *$ & $0.4683^{*}$ & $0.3568^{*}$ & $0.2870^{*}$ \\
\hline CO2eq11 & 1.1653 & 0.8489 & 0.7883 & 0.6738 & 0.5125 & 0.4118 \\
\hline CO2eq12 & 1.0868 & 0.6960 & 0.5935 & $0.4840^{*}$ & $0.3690 *$ & $0.2972^{*}$ \\
\hline CO3eq7 & 1.0429 & 1.0119 & 0.9963 & 0.9773 & 0.9339 & 0.9170 \\
\hline СO3eq9 & 1.2319 & 0.7663 & 0.6294 & 0.5211 & $0.3961^{*}$ & $0.3201^{*}$ \\
\hline CO3eq11 & 1.3272 & 0.9684 & 0.9002 & 0.7716 & 0.5874 & 0.4718 \\
\hline \multirow[t]{2}{*}{ CO3eq12 } & 1.2123 & 0.7817 & 0.6537 & 0.5460 & $0.4156^{*}$ & $0.3353^{*}$ \\
\hline & \multicolumn{6}{|c|}{ MAPE ratio } \\
\hline $\mathrm{AR}(1)$ & $0.9055 *$ & 0.9204 & 0.9266 & 0.9445 & 0.9677 & 0.9948 \\
\hline CO1eq7 & 1.0032 & 1.0003 & 1.0021 & 0.9960 & 0.9931 & 0.9899 \\
\hline CO1eq9 & 1.0033 & $0.7736^{*}$ & $0.7127^{*}$ & $0.6459 *$ & $0.5686^{*}$ & $0.5096 *$ \\
\hline CO1eq11 & 1.0153 & 0.8591 & 0.8137 & 0.7544 & 0.6645 & 0.5945 \\
\hline CO1eq12 & 0.9976 & $0.7710^{*}$ & $0.7075^{*}$ & $0.6406^{*}$ & $0.5631^{*}$ & $0.5049^{*}$ \\
\hline CO2eq7 & 0.9973 & 1.0077 & 1.0015 & 0.9925 & 0.9919 & 0.9818 \\
\hline CO2eq9 & 1.0723 & 0.8288 & 0.7483 & $0.6726^{*}$ & $0.5902^{*}$ & $0.5304^{*}$ \\
\hline CO2eq11 & 1.0981 & 0.9280 & 0.8798 & 0.8155 & 0.7176 & 0.6443 \\
\hline CO2eq12 & 1.0655 & 0.8349 & 0.7523 & $0.6721^{*}$ & $0.5888^{*}$ & $0.5295^{*}$ \\
\hline CO3eq7 & 1.0071 & 1.0097 & 1.0001 & 0.9905 & 0.9792 & 0.9670 \\
\hline СО3еq9 & 1.1379 & 0.8882 & 0.7840 & 0.7140 & $0.6266^{*}$ & $0.5627^{*}$ \\
\hline CO3eq11 & 1.1759 & 0.9945 & 0.9427 & 0.8759 & 0.7716 & 0.6920 \\
\hline CO3eq12 & 1.1281 & 0.8914 & 0.7847 & 0.7134 & $0.6248^{*}$ & $0.5609 *$ \\
\hline
\end{tabular}

Note: $\mathrm{CO} 1, \mathrm{CO} 2, \mathrm{CO} 3$ refer to the volatilities of the futures oil prices with 1-month, 2-months and 3-months maturities, whereas the equation number denotes the respective futures-based model. All MAPE and MSPE ratios have been normalized relative to the monthly no-change forecast. Bold face indicates predictive gains relatively to the no-change forecast. ${ }^{*}$ denotes that the model is among the set of the best performing models according to the Model Confidence Set (MCS) test. 
Table 3: Directional accuracy - full out-of-sample period. Evaluation period: 18/07/2007$05 / 08 / 2015$.

\begin{tabular}{|c|c|c|c|c|c|c|}
\hline Days ahead & 1-day & 5 -days & 10-days & 22-days & 44-days & 66-days \\
\hline & \multicolumn{6}{|c|}{ Directional accuracy } \\
\hline $\operatorname{AR}(1)$ & $0.6262 * *$ & $0.6570 * *$ & $0.6396 * *$ & $0.6327^{* *}$ & $0.6103^{* *}$ & $0.6013^{* *}$ \\
\hline CO1eq7 & 0.5408 & $0.6453^{* *}$ & $0.6224 * *$ & $0.6214^{* *}$ & $0.6075 * *$ & 0.5925 \\
\hline CO1eq9 & 0.5199 & $0.6632^{* *}$ & $0.6682^{* *}$ & $0.6716^{* *}$ & $0.6741 * *$ & $0.6786^{* *}$ \\
\hline CO1eq11 & 0.5234 & 0.5269 & 0.5264 & 0.5289 & 0.5284 & 0.5264 \\
\hline CO1eq12 & 0.5408 & $0.6587 * *$ & $0.6682^{* *}$ & $0.6692 * *$ & $0.6677^{* *}$ & $0.6731 * *$ \\
\hline $\mathrm{CO} 2 \mathrm{eq} 7$ & 0.5478 & $0.6418^{* *}$ & $0.6229 * *$ & $0.6194 * *$ & $0.6090 * *$ & 0.5940 \\
\hline CO2eq9 & $0.6308 * *$ & $0.6687^{* *}$ & $0.6751 * *$ & $0.6876^{* *}$ & $0.6905 * *$ & $0.6871^{* *}$ \\
\hline CO2eq11 & $0.6333^{* *}$ & $0.6338 * *$ & $0.6343 * *$ & $0.6348^{* *}$ & $0.6368 * *$ & $0.6333^{* *}$ \\
\hline CO2eq12 & $0.6308^{* *}$ & $0.6627 * *$ & $0.6672 * *$ & $0.6871^{* *}$ & $0.6900 * *$ & $0.6881 * *$ \\
\hline CO3eq7 & 0.5826 & $0.6413^{* *}$ & $0.6189 * *$ & $0.6234^{* *}$ & $0.6035^{* *}$ & 0.5826 \\
\hline CO3eq9 & $0.6313^{* *}$ & $0.6602 * *$ & $0.6796 * *$ & $0.6796 * *$ & $0.6851 * *$ & $0.6861 * *$ \\
\hline CO3eq11 & $0.6254^{* *}$ & $0.6249 *$ & $0.6254^{*}$ & $0.6229 * *$ & $0.6229 * *$ & $0.6249 * *$ \\
\hline CO3eq12 & $0.6348 * *$ & $0.6572 * *$ & $0.6711^{* *}$ & $0.6756^{* *}$ & $0.6821 * *$ & $0.6811^{* *}$ \\
\hline
\end{tabular}

Note: $\mathrm{CO} 1, \mathrm{CO} 2, \mathrm{CO} 3$ refer to the volatilities of the futures oil prices with 1-month, 2-months and 3-months maturities, whereas the equation number denotes the respective futures-based model. The statistical significance of the success ratios is tested based on the Pesaran and Timmermann (2009) under the null hypothesis of no directional accuracy. ${ }^{* *}$ and ${ }^{*}$ denote significance at $5 \%$ and $10 \%$ level, respectively. Bold face denotes improvement of the directional accuracy relatively to the no-change forecast. 
Table 4: Forecasting oil price volatility - Global Financial Crisis period. Evaluation period: 18/07/2007-31/07/2009.

\begin{tabular}{|c|c|c|c|c|c|c|}
\hline Days ahead & 1-day & 5-days & 10-days & 22-days & 44-days & 66 -days \\
\hline & \multicolumn{6}{|c|}{ MSPE ratio } \\
\hline $\operatorname{AR}(1)$ & $0.7410^{*}$ & 0.7893 & 0.8385 & 0.8764 & 0.8674 & 0.9153 \\
\hline CO1eq7 & 0.9250 & 0.8489 & 0.8517 & 0.8138 & 0.7626 & 0.7228 \\
\hline CO1eq9 & 0.8611 & $0.5260^{*}$ & $0.4984^{*}$ & $0.3978^{*}$ & $0.2558^{*}$ & $0.1851^{*}$ \\
\hline CO1eq11 & 0.9530 & 0.7725 & 0.7308 & 0.5799 & 0.3687 & 0.2678 \\
\hline CO1eq12 & 0.8445 & $0.5154^{*}$ & $0.4817^{*}$ & $0.3843^{*}$ & $0.2466^{*}$ & $0.1783^{*}$ \\
\hline CO2eq7 & 0.8978 & 0.8592 & 0.8448 & 0.7913 & 0.7338 & 0.6911 \\
\hline CO2eq9 & 0.8454 & 0.5539 & $0.4890 *$ & $0.3736^{*}$ & $0.2370^{*}$ & $0.1717^{*}$ \\
\hline CO2eq11 & 0.9396 & 0.7636 & 0.7212 & 0.5716 & 0.3634 & 0.2641 \\
\hline CO2eq12 & 0.8322 & 0.5818 & 0.5152 & $0.3964^{*}$ & $0.2526^{*}$ & $0.1828^{*}$ \\
\hline CO3eq7 & 0.9362 & 0.8865 & 0.8460 & 0.7888 & 0.7051 & 0.6510 \\
\hline СO3eq9 & 0.9555 & 0.6125 & 0.5383 & 0.4228 & $0.2678^{*}$ & $0.1948^{*}$ \\
\hline CO3eq11 & 1.0578 & 0.8597 & 0.8127 & 0.6460 & 0.4108 & 0.2971 \\
\hline \multirow[t]{2}{*}{ CO3eq12 } & 0.9366 & 0.6334 & 0.5752 & 0.4584 & $0.2917^{*}$ & $0.2115^{*}$ \\
\hline & \multicolumn{6}{|c|}{ MAPE ratio } \\
\hline $\mathrm{AR}(1)$ & $0.9055^{*}$ & 0.9204 & 0.9266 & 0.9445 & 0.9677 & 0.9948 \\
\hline CO1eq7 & 0.9252 & 0.9062 & 0.9164 & 0.8824 & 0.8514 & 0.8193 \\
\hline CO1eq9 & 0.9146 & $0.7060^{*}$ & $0.6669 *$ & $0.5905^{*}$ & $0.4788^{*}$ & $0.3944^{*}$ \\
\hline CO1eq11 & 0.9408 & 0.8274 & 0.7852 & 0.6979 & 0.5684 & 0.4688 \\
\hline CO1eq12 & 0.9048 & $0.6979 *$ & $0.6548^{*}$ & $0.5800^{*}$ & $0.4705^{*}$ & $0.3875^{*}$ \\
\hline CO2eq7 & 0.9274 & 0.9159 & 0.9221 & 0.8771 & 0.8454 & 0.8006 \\
\hline CO2eq9 & 0.9485 & 0.7519 & 0.6782 & 0.5921 & $0.4757^{*}$ & 0.3921 \\
\hline CO2eq11 & 0.9917 & 0.8738 & 0.8266 & 0.7337 & 0.5959 & 0.4922 \\
\hline CO2eq12 & 0.9379 & 0.7630 & 0.6903 & 0.5988 & $0.4820^{*}$ & 0.3968 \\
\hline CO3eq7 & 0.9451 & 0.9355 & 0.9127 & 0.8752 & 0.8285 & 0.7796 \\
\hline СО3еq9 & 1.0076 & 0.7973 & 0.7107 & 0.6343 & 0.5124 & $0.4222^{*}$ \\
\hline CO3eq11 & 1.0496 & 0.9256 & 0.8772 & 0.7804 & 0.6350 & 0.5220 \\
\hline CO3eq12 & 0.9958 & 0.8062 & 0.7188 & 0.6436 & 0.5201 & $0.4277^{*}$ \\
\hline
\end{tabular}

Note: $\mathrm{CO} 1, \mathrm{CO} 2, \mathrm{CO} 3$ refer to the volatilities of the futures oil prices with 1-month, 2-months and 3-months maturities, whereas the equation number denotes the respective futures-based model. All MAPE and MSPE ratios have been normalized relative to the monthly no-change forecast. Bold face indicates predictive gains relatively to the no-change forecast. ${ }^{*}$ denotes that the model is among the set of the best performing models according to the Model Confidence Set (MCS) test. 
Table 5: Directional accuracy - Global Financial Crisis period. Evaluation period: 18/07/2007$31 / 07 / 2009$.

\begin{tabular}{|c|c|c|c|c|c|c|}
\hline Days ahead & 1-day & 5 -days & 10-days & 22-days & 44-days & 66-days \\
\hline & \multicolumn{6}{|c|}{ Directional accuracy } \\
\hline $\operatorname{AR}(1)$ & $0.6385^{* *}$ & $0.6690 * *$ & 0.5892 & 0.5962 & 0.5446 & 0.5188 \\
\hline CO1eq7 & 0.5305 & 0.5211 & 0.5164 & 0.5469 & 0.5282 & 0.5446 \\
\hline CO1eq9 & 0.5047 & $0.8779 * *$ & $0.8592 * *$ & $0.8732^{* *}$ & $0.8873^{* *}$ & $0.9343^{* *}$ \\
\hline CO1eq11 & 0.4554 & $0.7981 * *$ & $0.7817 * *$ & $0.7911 * *$ & $0.7887 * *$ & $0.8333^{* *}$ \\
\hline CO1eq12 & 0.4812 & $0.8615 * *$ & $0.8592 * *$ & $0.8638 * *$ & $0.8850 * *$ & $0.9343^{* *}$ \\
\hline CO2eq7 & 0.5329 & 0.5188 & 0.5000 & 0.5493 & 0.5399 & 0.5376 \\
\hline CO2eq9 & $0.6432 * *$ & $0.6878 * *$ & $0.7230 * *$ & $0.7653^{* *}$ & $0.7817 * *$ & $0.8615 * *$ \\
\hline CO2eq11 & $0.6221 * *$ & $0.6479 * *$ & $0.6878 * *$ & $0.7230 * *$ & $0.7324 * *$ & $0.7371^{* *}$ \\
\hline CO2eq12 & $0.6432 * *$ & $0.7019 * *$ & $0.7207 * *$ & $0.7746 * *$ & $0.7676^{* *}$ & $0.8474^{* *}$ \\
\hline CO3eq7 & 0.5704 & 0.5610 & 0.5516 & 0.5822 & 0.5634 & 0.5610 \\
\hline CO3eq9 & $0.6291 * *$ & $0.6831 * *$ & $0.7207 * *$ & $0.7465 * *$ & $0.7676^{* *}$ & $0.8286 * *$ \\
\hline CO3eq11 & $0.6291 * *$ & $0.6667 * *$ & $0.6549 *$ & $0.6432^{* *}$ & $0.6362^{*}$ & $0.7934^{* *}$ \\
\hline CO3eq12 & $0.6338^{* *}$ & $0.6831 * *$ & $0.7230 * *$ & $0.7465 * *$ & $0.7676^{* *}$ & $0.8263^{* *}$ \\
\hline
\end{tabular}

Note: $\mathrm{CO} 1, \mathrm{CO} 2, \mathrm{CO} 3$ refer to the volatilities of the futures oil prices with 1-month, 2-months and 3-months maturities, whereas the equation number denotes the respective futures-based model. The statistical significance of the success ratios is tested based on the Pesaran and Timmermann (2009) under the null hypothesis of no directional accuracy. ${ }^{* *}$ and ${ }^{*}$ denote significance at $5 \%$ and $10 \%$ level, respectively. Bold face denotes improvement of the directional accuracy relatively to the no-change forecast. 
Table 6: Forecasting oil price volatility - Oil Price Collapse period. Evaluation period: 19/06/2014-05/08/2015.

\begin{tabular}{|c|c|c|c|c|c|c|}
\hline Days ahead & 1-day & 5-days & 10-days & 22-days & 44-days & 66 -days \\
\hline & \multicolumn{6}{|c|}{ MSPE ratio } \\
\hline $\operatorname{AR}(1)$ & $0.9257^{*}$ & 0.8652 & 0.8755 & 0.9203 & 0.9435 & 0.9525 \\
\hline CO1eq7 & 1.0010 & 1.0070 & 0.9912 & 0.9703 & 0.9469 & 0.9195 \\
\hline CO1eq9 & 1.0023 & $0.4986 *$ & $0.4106^{*}$ & $0.3413^{*}$ & $0.2885^{*}$ & $0.1893^{*}$ \\
\hline CO1eq11 & 1.0103 & 0.5717 & 0.4748 & 0.3954 & 0.3924 & $0.2194^{*}$ \\
\hline CO1eq12 & 0.9956 & $0.4138^{*}$ & $0.3438^{*}$ & $0.3438 *$ & $0.2898^{*}$ & $0.1902^{*}$ \\
\hline CO2eq7 & 0.9613 & 0.9938 & 0.9439 & 0.9372 & 0.9335 & 0.9156 \\
\hline CO2eq9 & 1.3885 & 0.6124 & 0.5033 & $0.4064^{*}$ & $0.3423^{*}$ & $0.2298^{*}$ \\
\hline CO2eq11 & 1.3833 & 0.7627 & 0.6363 & 0.5296 & 0.4460 & $0.2941^{*}$ \\
\hline CO2eq12 & 1.3802 & 0.6210 & 0.5132 & $0.4121^{*}$ & $0.3463^{*}$ & $0.2314^{*}$ \\
\hline CO3eq7 & 1.0049 & 0.9879 & 0.9463 & 0.9376 & 0.9098 & 0.9127 \\
\hline CO3eq9 & 1.6997 & 0.7759 & 0.5301 & 0.4997 & 0.4043 & $0.2724^{*}$ \\
\hline CO3eq11 & 1.7083 & 0.9552 & 0.8018 & 0.6689 & 0.5656 & 0.3686 \\
\hline \multirow[t]{2}{*}{ CO3eq12 } & 1.6895 & 0.7835 & 0.5450 & 0.4925 & 0.4268 & $0.2849 *$ \\
\hline & \multicolumn{6}{|c|}{ MAPE ratio } \\
\hline $\operatorname{AR}(1)$ & $0.9055^{*}$ & 0.9204 & 0.9266 & 0.9445 & 0.9677 & 0.9948 \\
\hline CO1eq7 & 1.0104 & 1.0033 & 0.9999 & 0.9916 & 0.9688 & 0.9591 \\
\hline CO1eq9 & 1.0041 & $0.6682^{*}$ & $0.6309 *$ & $0.5565 *$ & $0.4684^{*}$ & $0.3874^{*}$ \\
\hline CO1eq11 & 1.0127 & 0.7323 & 0.6953 & 0.6144 & 0.5260 & $0.4237^{*}$ \\
\hline CO1eq12 & 1.0008 & $0.6707^{*}$ & $0.6318^{*}$ & $0.5567^{*}$ & $0.4667^{*}$ & $0.3860^{*}$ \\
\hline CO2eq7 & 0.9926 & 1.0009 & 0.9820 & 0.9823 & 0.9673 & 0.9639 \\
\hline CO2eq9 & 1.1587 & 0.7562 & 0.7015 & $0.6059 *$ & $0.5172^{*}$ & $0.4334^{*}$ \\
\hline CO2eq11 & 1.1485 & 0.8216 & 0.7851 & 0.6931 & 0.5904 & 0.4902 \\
\hline CO2eq12 & 1.1578 & 0.7619 & 0.7050 & $0.6059 *$ & $0.5157^{*}$ & $0.4315^{*}$ \\
\hline CO3eq7 & 1.0398 & 0.9921 & 0.9954 & 0.9907 & 0.9571 & 0.9522 \\
\hline СO3eq9 & 1.3079 & 0.8627 & 0.7451 & 0.6721 & 0.5734 & $0.4729 *$ \\
\hline CO3eq11 & 1.3182 & 0.9528 & 0.9054 & 0.7985 & 0.6830 & 0.5581 \\
\hline CO3eq12 & 1.3062 & 0.8670 & 0.7551 & 0.6808 & 0.5806 & $0.4768^{*}$ \\
\hline
\end{tabular}

Note: $\mathrm{CO} 1, \mathrm{CO} 2, \mathrm{CO} 3$ refer to the volatilities of the futures oil prices with 1-month, 2-months and 3-months maturities, whereas the equation number denotes the respective futures-based model. All MAPE and MSPE ratios have been normalized relative to the monthly no-change forecast. Bold face indicates predictive gains relatively to the no-change forecast. ${ }^{*}$ denotes that the model is among the set of the best performing models according to the Model Confidence Set (MCS) test. 
Table 7: Directional accuracy - Oil Price Collapse period. Evaluation period: 19/06/2014$05 / 08 / 2015$.

\begin{tabular}{|c|c|c|c|c|c|c|}
\hline Days ahead & 1-day & 5 -days & 10-days & 22-days & 44-days & 66-days \\
\hline & \multicolumn{6}{|c|}{ Directional accuracy } \\
\hline $\operatorname{AR}(1)$ & $0.5973^{*}$ & 0.5664 & $0.6150 * *$ & 0.5442 & 0.4735 & 0.3805 \\
\hline CO1eq7 & 0.5000 & 0.5088 & 0.5044 & 0.4469 & 0.4336 & 0.4149 \\
\hline CO1eq9 & 0.5044 & $0.8805 * *$ & $0.9159 * *$ & $0.9248 * *$ & $0.9336 * *$ & $0.9602^{* *}$ \\
\hline CO1eq11 & 0.4779 & $0.7920 * *$ & $0.8319 * *$ & $0.8186 * *$ & $0.9027 * *$ & $0.8982^{* *}$ \\
\hline CO1eq12 & 0.4735 & $0.8628 * *$ & $0.8761 * *$ & $0.9336 * *$ & $0.9425 * *$ & $0.9602^{* *}$ \\
\hline CO2eq7 & 0.5133 & 0.5132 & 0.5354 & 0.4690 & 0.4204 & 0.4115 \\
\hline CO2eq9 & $0.6062 * *$ & $0.6549 * *$ & $0.7876^{* *}$ & $0.8009 * *$ & $0.8982 * *$ & $0.9159 * *$ \\
\hline CO2eq11 & $0.6062 * *$ & 0.5841 & $0.7655 * *$ & $0.7832 * *$ & $0.8496 * *$ & $0.8628 * *$ \\
\hline CO2eq12 & $0.6062 * *$ & $0.6726^{* *}$ & $0.7876^{* *}$ & $0.8230 * *$ & $0.8894 * *$ & $0.9027 * *$ \\
\hline CO3eq7 & 0.4867 & 0.4956 & 0.5088 & 0.4425 & 0.4292 & 0.4381 \\
\hline CO3eq9 & $0.6195 * *$ & $0.5973^{*}$ & $0.7522 * *$ & $0.7301 * *$ & $0.8628 * *$ & $0.8982^{* *}$ \\
\hline CO3eq11 & $0.6106 * *$ & 0.5752 & $0.6637^{*}$ & $0.6504^{* *}$ & $0.6991 * *$ & $0.8230 * *$ \\
\hline CO3eq12 & $0.6283^{* *}$ & $0.6106 * *$ & $0.7655^{* *}$ & $0.7876 * *$ & $0.8761 * *$ & $0.8805^{* *}$ \\
\hline
\end{tabular}

Note: $\mathrm{CO} 1, \mathrm{CO} 2, \mathrm{CO} 3$ refer to the volatilities of the futures oil prices with 1-month, 2-months and 3-months maturities, whereas the equation number denotes the respective futures-based model. The statistical significance of the success ratios is tested based on the Pesaran and Timmermann (2009) under the null hypothesis of no directional accuracy. ${ }^{* *}$ and ${ }^{*}$ denote significance at $5 \%$ and $10 \%$ level, respectively. Bold face denotes improvement of the directional accuracy relatively to the no-change forecast. 